\title{
Bleeding Complications in Atrial Fibrillation Patients on Anticoagulant Therapy
}

\author{
IONELA SILIVASTRU (COZLEA) ${ }^{1}$, GABRIELA KERESZTESI ${ }^{1}$, ARTHUR ATILLA KERESZTESI ${ }^{2}$, DANIEL LAURENTIU COZLEA ${ }^{1,3 *}$, \\ CARMEN CALDARARU ${ }^{3,4}$, DANIELA ECATERINA DOBRU 3,5 \\ ${ }^{1}$ Emergency Clinical County Hospital, 50 Gh. Marinescu Str., 540136, Tirgu Mures, Romania \\ IInstitute of Forensic Medicine, 38 Gh. Marinescu Str., 540139, Tirgu Mures, Romania \\ 3University of Medicine, Pharmacy, Sciences and Technology of Tirgu Mures, Faculty of Medicine, Department M3,50 Gh. \\ Marinescu Str., 540136, Tirgu Mures, Romania \\ ${ }^{4}$ Clinical County Hospital, Medical Department, 50 Gh. Marinescu Str., 540136, Tirgu Mures, Romania \\ ${ }^{5}$ Clinical County Hospital, Gastroenterology Department, 50 Gh. Marinescu Str., 540136, Tirgu Mures, Romania
}

\begin{abstract}
The risk of bleeding in atrial fibrillation patients on direct oral anticoagulant treatment increases with age; particularly dabigatran is associated with a higher risk of gastrointestinal bleeding in elderly patients, low body mass $(<48 \mathrm{~kg})$ and women due to the induced dyspepsia. We aimed to evaluate the safety of direct oral anticoagulants (DOAC) dabigatran, rivaroxaban and apixaban by comparing each agent with a widely used vitamin $K$ antagonist (VKA) - acenocoumarol in terms of bleeding event rates. A retrospective study regarding bleeding events in atrial fibrillation patients treated with oral anticoagulation (OAC) was performed. Haematuria, epistaxis and haemoptysis were considered minor events and intracranial bleeding, gastrointestinal bleeding (superior or inferior), blood transfusion after haemorrhagic events linked to OAC treatment were considered to be major events. A number of 219 atrial fibrillation patients were included using electronic medical records: 118 patients treated with DOAC's (82 using dabigatran, 28 on rivaroxaban regimen and 8 cases treated with apixaban) and 101 cases had vitamin $K$ antagonists' treatment (acenocumarol). A total of 75 bleeding events were encountered ( 70 minor and 5 major). A higher number of events were encountered in patients treated with DOAC's, but with no statistically significant difference compared to acenocumarol. The associated risk factors did not play a decisive role in bleeding events in the two treatment groups. No statistical significant difference was noted between the occurrence of haemorrhagic events and the class of oral anticoagulant treatment used (DOAC vs. VKA).
\end{abstract}

Keywords: atrial fibrillation, bleeding events, direct oral anticoagulants, vitamin $K$ antagonist

The main concern regarding atrial fibrillation (AF) patients are thromboembolic events, a problem for each physician. To prevent such events and to improve survival, oral anticoagulant (OAC) agents are used, either classic ones or part of the new generation of anticoagulants.

The use of direct oral anticoagulant agents (DOAC), such as dabigatran, rivaroxaban or apixaban is contraindicated in certain conditions such as severe chronic kidney disease (creatinine clearance $<15 \mathrm{ml} / \mathrm{min}$ ) or hepatic disease with coagulopathy, therefore vitamin K antagonists (VKA) could be used as an alternative. [1] .

The risk of bleeding in AF patients on DOAC increases with age, with reports in particular for dabigatran which has been associated with a higher risk of gastrointestinal bleeding (GI) in elderly patients, low body mass (<48 kg) and women due to the induced dyspepsia $[2,3]$.

A meta-analysis including over 70,000 patients on direct anticoagulants and vitamin $\mathrm{K}$ antagonists (warfarin) suggests that the DOAC significantly reduce intracranial haemorrhage, but increase gastrointestinal bleeding [4]. One study focuses on the outcomes of the bleeding, especially the major events, once they occur [5]. The results indicate that DOAC patients had lower death rates after major intracranial bleeding events, compared to warfarin.

Regarding the GI bleeding events, minor or major, studies suggest a higher number of events in the DOAC patients compared to warfarin treatment, especially among patients aged 75 years or over [6].

In rivaroxaban use, the most frequent minor events described are mucosal haemorrhage (epistaxis, gum bleeding, and haematuria) and secondary anaemia, with recommendations to monitoring haemoglobin and haematocrit levels. Regarding the alcohol intake while using DOACs, this should be moderated [8].

The objective of the present study was to evaluate the bleeding event rates in patients treated with DOAC's (dabigatran, rivaroxaban and apixaban) compared to patients using a vitamin $\mathrm{K}$ antagonist - acenocoumarol.

The protocol of this study was approved by the Ethics Committee of the University of Medicine and Pharmacy of Tirgu Mures, Romania, in accordance with standards of 1964 Helsinki declaration and its later amendments or comparable ethical standards.

\section{Experimental part \\ Methods}

A retrospective observational study on consecutive 219 adult atrial fibrillation out- and in patients treated with OAC was performed.

The considered duration of research was between 2013, July, 1-2016, J une, 30, irrespectively of the time when anticoagulation was started or interrupted by various reasons (other side-effects than GI bleeding, switching on other treatment, non-compliance, discontinuation, and death).

Documented presence of AF (medical history, physical examination, restEKG and Holter recordings) was the main inclusion criterion in the study, irrespectively of its etiology. Other related conditions (presence of heart failure, systemic hypertension, anaemia, diabetes mellitus, and alcohol abuse) were explored. All patients signed informed consent.

\footnotetext{
* email: cozlead@yahoo.com, Phone: +40.742.26.44.32
} 
Patients were divided in two groups: group I: 118 patients receiving DOAC's ( 82 on dabigatran, 28 on rivaroxaban and 8 on apixaban), respectively group II of 101 cases with VKA regimen.

Anticoagulation was started and conducted using ESC/ EHRA guidelines. We considered the approved regimens for the DOAC: dabigatran $150 \mathrm{mg}$ b.i.d, rivaroxaban $20 \mathrm{mg}$ o.d., or apixaban $5 \mathrm{mg}$ b.i.d..

In group II, acenocoumarol dose was adjusted to achieve an INR target between 2.5-3. Mean period of anticoagulation was 32 months.

All included patients had a serum creatinine levels clearance over $20 \mathrm{~mL} / \mathrm{min}$ (MDRD formula).

For haemorrhagic events four class classification of bleeding provided by American College of Surgeons' advanced trauma life support (ATLS) was used.

Bleeding events occurred during the 42 months of treatment were divided in

-minor events including hospitalization for haematuria, epistaxis, haemoptysis;

-major events including hospitalization for intracranial bleeding, GI bleeding (superior or inferior), and blood transfusion after haemorrhagic events linked to OAC treatment.

\section{Statistical analysis}

Patient data were included in to a Microsoft Excel 2010 file (Microsoft ${ }^{\circ}$, Redmond, WA, USA); Epi Info version 3.5.3 (CDC Epi Info ${ }^{\text {TM }} 3$, Atlanta, GE, USA) software was used for statistical analysis. Continuous variables are reported as mean \pm standard deviation (SD) and categorical variables as observed number of patients. Statistical significance value was set at $p<0.05$.

In the period 01.07.2013 - 30.06.2016 the electronic registries were consulted and each patient was checked for the occurrence of haemorrhagic events.

\section{Results and discussions}

At baseline, the anticoagulant treatment administered to the patients was as follows: 118 patients $(53.8 \%)$ had direct oral anticoagulant treatment: 82 patients (37.44\%) had dabigatran, 28 patients (12.78\%) rivaroxaban and 8 patients (3.65\%) apixaban; 101 patients (46.2\%) received vitamin $\mathrm{K}$ antagonist (acenocumarol), with regular INR check.

Regarding the concomitant diseases, heart failure and arterial hypertension were the most frequent comorbidities. (fig. 1)

The mean age of all the patients at baseline was $71 \pm 9$ years; no statistical significance was observed between the mean age of the patients and the oral anticoagulant treatment administered (DOAC and acenocumarol). Regarding the sex of the patients, 97 were male and 122

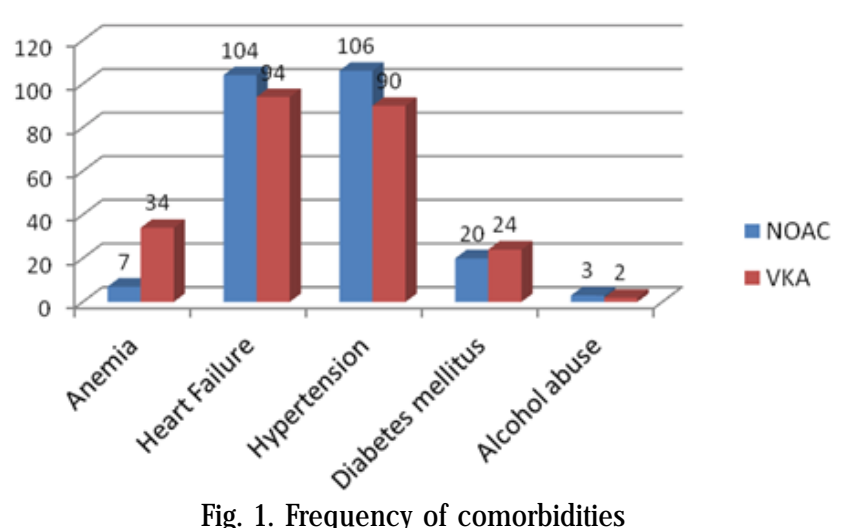

females, no statistically significant result was observed between the sex distribution and OAC treatment.

The mean period of DOAC treatment for all patients was 32 months (dabigatran group), 21 months (rivaroxaban group), 12 months (apixaban group) and 40 months on acenocumarol-treated patients.

At the time of the electronic register check of all the patients, $31.96 \%$ of patients (70 cases) had presented minor bleeding events and 5 patients presented major haemorrhagic events (fig. 2).

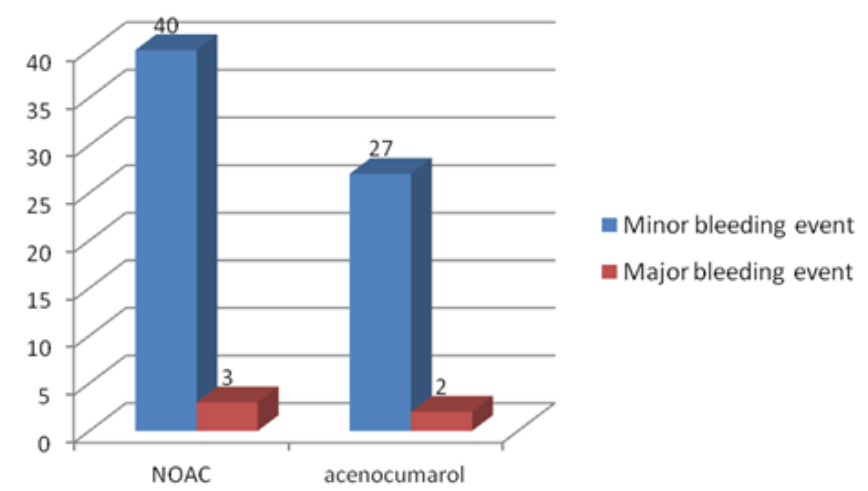

Fig. 2. Anticoagulant treatment class and types of haemorrhagic events

The results show no statistically significant difference irrespectively of the class of oral anticoagulant regimen and the number of haemorrhagic events (Chi-square uncorrected $p=0.6499$ ).

Regarding the number of minor and major bleeding events, the $p$ value was $>0.05$, with no statistical significance between the two types of anticoagulation regimens (VKA vs DOAC) (table 1 ).

Table 1

CONTINGENCY TABLE BETWEEN THE ORAL ANTICOAGULANT TREATMENT APPLIED AND THE PRESENCE OF BLEEDING EVENTS

\begin{tabular}{|l|c|c|c|}
\hline & $\begin{array}{c}\text { With } \\
\text { bleedings }\end{array}$ & $\begin{array}{c}\text { Without } \\
\text { bleedings }\end{array}$ & $\begin{array}{c}P \\
\text { value }\end{array}$ \\
\hline Acenocumarol & 33 & 68 & \multirow{2}{*}{0.6499} \\
\hline DOAC & 42 & 76 & \\
\hline
\end{tabular}

A total number of 40 patients treated with DOAC's experienced minor haemorrhagic events (40 out of 118 studied cases). Hematuria was found in 38 cases ( 30 dabigatran-related, 8 patients treated with rivaroxaban) and epistaxis in two dabigatran-treated patients.

Regarding the major bleeding events, 3 patients were using DOAC's (2 bleedings dabigatran-related and one linked to rivaroxaban treatment) and 2 patients were treated with acenocumarol, no statistically significant difference between the two treatments of OACs $(p=0.6638$, Fisher's exact test). Out of the 5 cases, 3 patients presented upper GI bleeding (one with need of blood transfusion) and two patients presented lower $\mathrm{Gl}$ bleeding.

None of the associated diseases correlated statistically with occurrence of minor or major bleeding events, but there was a higher prevalence of heart failure and hypertension among the patients with major haemorrhage (Gl bleeding).

The major elements that have a proven additional role in bleeding events (alcohol, anemia) were ruled out in the present study, hence the discussion on the subject.

We also found that a higher number of patients presenting minor bleeding events were using dabigatran. 


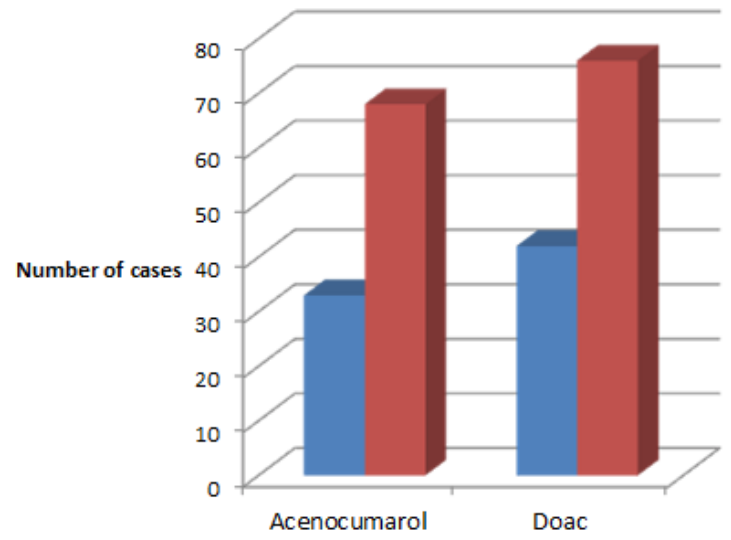

Fig. 3. The oral anticoagulant treatment applied and the presence of bleeding event

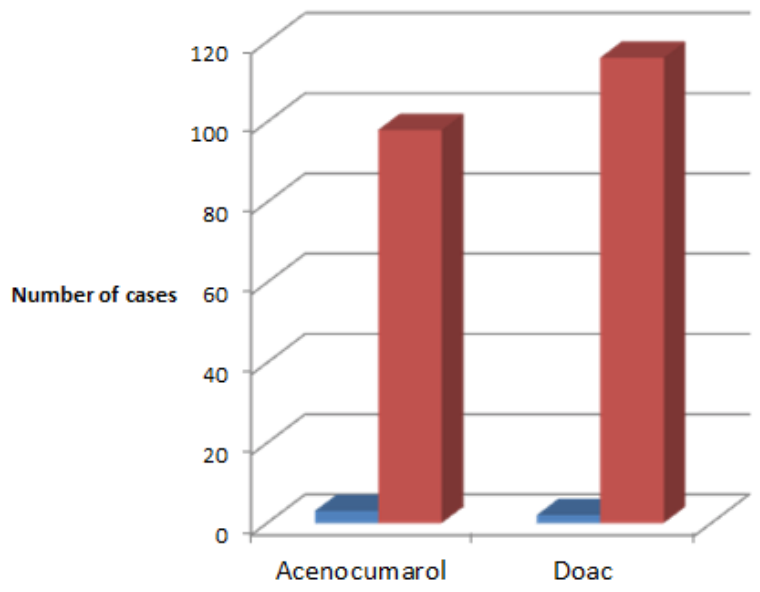

Fig. 4. Major bleeding events

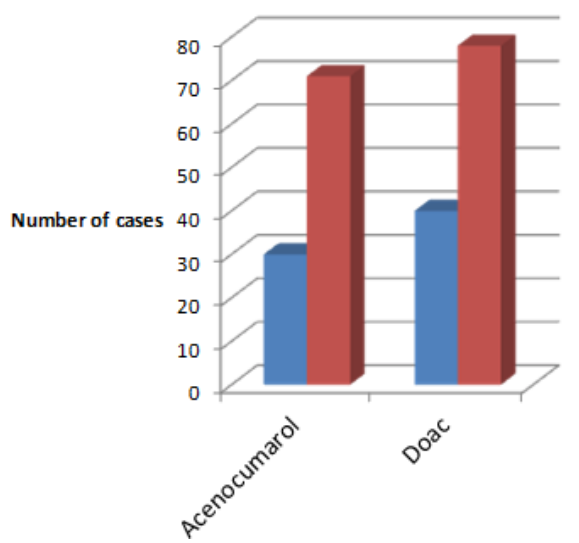

Fig. 5. Minor bleeding events

In one similar study, but on a smaller group of patients [9], the authors found that dabigatran had more frequently gastrointestinal symptom like nausea or gastritis, while rivaroxaban caused more frequently minor bleedings. We also found that a higher number of patients presenting minor bleeding events were on dabigatran, but with no statistical significance.

Anticoagulant treatment with dabigatran, rivaroxaban or apixaban, are considered first-line agents for treatment and prophylaxis of venous thromboembolic disease with many ongoing randomized multicentre studies [10]. Recent data results suggest rivaroxaban and apixaban are the most promising DOAC agents, with non-inferior results compared to warfarin in pulmonary embolism treatment
Table 2

MAJ OR BLEEDING EVENTS

\begin{tabular}{|l|c|c|c|}
\hline & $\begin{array}{c}\text { With major } \\
\text { haemorrhage }\end{array}$ & $\begin{array}{c}\text { Without } \\
\text { haemorrhage }\end{array}$ & P value \\
\hline Acenocumarol & 3 & 98 & \multirow{2}{*}{0.6638} \\
\hline DOAC & 2 & 116 & \\
\hline
\end{tabular}

With major haemorrhage

without major haemorrhage
Table 3

MINOR BLEEDING EVENTS

\begin{tabular}{|l|c|c|c|}
\hline & $\begin{array}{c}\text { With minor } \\
\text { haemorrhage }\end{array}$ & $\begin{array}{c}\text { Without minor } \\
\text { haemorrhage }\end{array}$ & P value \\
\hline Acenocumarol & 30 & 71 & \multirow{2}{*}{0.5069} \\
\hline DOAC & 40 & 78 & \\
\hline
\end{tabular}

[11-13]. In patients with hepatic dysfunctions dabigatran may be the bestNOAC option or for those using drugs which can interfere with CYP3A4 activity; Although specific methods for determining plasma concentrations of apixaban and rivaroxaban are described in literature, till now they are no widely used in general practice in terms of monitoring therapeutic regimen due to relative highcosts and specific laboratory equipment $[14,15]$.

\section{Conclusions}

No statistical difference was noted between the occurrence of haemorrhagic events and the class of oral anticoagulant treatment used (DOAC vs. VKA).

Hematuria was the most frequent minor bleeding event in the DOAC group of patients.

Upper gastrointestinal bleeding was the most frequent major haemorrhagic event, with majority of the patients on direct anticoagulant treatment.

\section{References}

1.HEIDBUCHEL $H$, VERHAMME P, ALINGS M, ANTZ M, DIENER HC, HACKE W etal. Updated European Heart Rhythm Association practical guide on the use of non-vitamin-K antagonist anticoagulants in patients 
with non-valvular atrial fibrillation: Executive summary. Eur Heart J. 2017;38(27):2137-2149.

2.KUNDU A, SARDAR P, CHATTERJEE S, ARONOW WS, OWAN T, RYAN J). Minimizing the risk of bleeding with NOACs in the elderly. Drugs Aging.2016;33(7):491-500.

3.YAMASHITA T, WATANABE E, IKEDA T, SHIGA T, KUSANO KF, TAKAHASHI N, et al. Observational study of the effects of dabigatran on gastrointestinal symptoms in patients with non-valvular atrial fibrillation. J Arrhythm. 2014; 30(6):478-484.

4.RUFF CT, GIUGLIANO RP, BRAUNWALD E, HOFFMAN EB, DEENADAYALU N, EZEKOWITZ MD, et al. Comparison of the efficacy and safety of new oral anticoagulants with warfarin in patients with atrial fibrillation: a meta-analysis of randomised trials. Lancet.2014;383(9921):955-62.

5.SKAISTIS J, TAGAMI T. Risk of fatal bleeding in episodes of major bleeding with new oral anticoagulants and vitamin K antagonists: A systematic review and meta-analysis. PLoS One. 2015;10(9): e0137444. 6.HALPERIN J L, HANKEY GJ , WOJ DYLA DM, PICCINI JP, LOKHNYGINA $Y$, PATEL MR et al. Efficacy and safety of rivaroxaban compared with warfarin among elderly patients with nonvalvular atrial fibrillation in the Rivaroxaban Once Daily, Oral, DirectFactor Xa Inhibition Compared With Vitamin K Antagonism for Prevention of Stroke and Embolism Trial in Atrial Fibrillation (ROCKET AF). Circulation 2014;130:138-46. 7.LIP GYH, LANE DA. Matching the NOAC to the patient: remember the modifiable bleeding risk factors. J Am CollCardiol. 2015;66(21):2282-2284.

8.SHERWOOD M, NESSEL C, HELLKAMP A, MAHAFFEY KW, PICCINI $J P$, SUH EY et al. Gastrointestinal bleeding in patients with atrial fibrillation treated with rivaroxaban or warfarin.: ROCKET AF Trial.J Am CollCardiol2015; 66(21):2271-2281.
9.GORZELAK-PABICE P, DURAJ I, SZLAGOWSKA L, CIASTKOWSKA A, BRONCEL M. Comparison of the safety of rivaroxaban versus dabigatran therapy in patients with persistent atrial fibrillation. Pol MerkurLekarski.2014;37(221):261-264.

10.COULIS AA, MACKEY WC. A Review of the Efficacy and Safety Profiles of the Novel Oral Anticoagulants in the Treatment and Prevention of Venous Thromboembolism.ClinTher. 2018 pii: S01492918(18)30504-6. doi: 10.1016/j.clinthera.2018.10.009. [Epub ahead of print]

11.*** The EINSTEIN-PE Investigators, BULLER HR, PRINS MH, LENSIN AW, DECOUSUS H, J ACOBSON BF, MINAR E et al. Oral rivaroxaban for the treatment of symptomatic pulmonary embolism. N Engl J Med 2012; 366(14):1287-1297.

12.JUNG H, MATEI D, HECSER LM, BOHNERT, S. POLLAK. Pulmonary pathology in drug related deaths. Rom J Legal Med 2007;15(2):83-90. 13.RUDD KM, PHILLIPS EL. New oral anticoagulants in the treatment of pulmonary embolism: efficacy, bleeding risk, and monitoring. Thrombosis 2013; 2013:973710.

14.TILEA I, POPA DANIELA S, SZAKACS XANTUS T, PRIMEJ DIE D, GRIGORESCU B, T\&ILEA B, BOCICOR AE, VARGA A. Determination of Apixaban Levels in Human Plasma by a High-Throughput Liquid Chromatographic Tandem Mass Spectrometry Assay. Rev. Romana Med. Lab, 2015, 23(1):115-125

15. VARGA A, SERBAN RC, MUNTEAN DL, TATAR CM, FARCZADI L, TILEA I. Rapid liquid chromatography tandem mass spectrometry determination of rivaroxaban levels in human plasma for therapeutic drug monitoring. Rev. Romana Med. Lab, 2017, 25(2):145-155

Manuscript received: 6.12 .2018 